\title{
Independent and interactive effects of DOF affecting germination 1 (DAG1) and the Della proteins GA insensitive (GAl) and Repressor of ga1-3 (RGA) in embryo development and seed germination
}

\author{
Alessandra Boccaccini ${ }^{1 \dagger}$, Silvia Santopolo ${ }^{1 \dagger}$, Davide Capauto ${ }^{1}$, Riccardo Lorrai ${ }^{1}$, Emanuele Minutello ${ }^{1}$, \\ Katia Belcram ${ }^{2}$, Jean-Cristophe Palauqui ${ }^{2}$, Paolo Costantino ${ }^{1}$ and Paola Vittorioso ${ }^{1,3^{*}}$
}

\begin{abstract}
Background: The transcription factor DOF AFFECTING GERMINATION1 (DAG1) is a repressor of seed germination acting downstream of the master repressor PHYTOCROME INTERACTING FACTOR3-LIKE 5 (PIL5). Among others, PIL5 induces the expression of the genes encoding the two DELLA proteins GA INSENSITIVE 1 (GAI) and REPRESSOR OF ga1-3 (RGA).

Results: Based on the properties of gai-t6 and rga28 mutant seeds, we show here that the absence of RGA severely increases dormancy, while lack of GAl only partially compensates $R G A$ inactivation. In addition, the germination properties of the dag1rga28 double mutant are different from those of the dag1 and rga28 single mutants, suggesting that RGA and DAG1 act in independent branches of the PIL5-controlled germination pathway. Surprisingly, the daglgai-t6 double mutant proved embryo-lethal, suggesting an unexpected involvement of (a possible complex between) DAG1 and GAI in embryo development.
\end{abstract}

Conclusions: Rather than overlapping functions as previously suggested, we show that RGA and GAl play distinct roles in seed germination, and that GAI interacts with DAG1 in embryo development.

Keywords: DAG1, GAl, RGA, Seed germination, Embryogenesis, Arabidopsis thaliana

\section{Background}

Seed germination is controlled by multiple endogenous and environmental factors [1], which are integrated to trigger this developmental process at the right time. Two plant hormones play important roles in seed germination: gibberellins (GA), which have an inductive effect, and abscissic acid (ABA), which inhibits the process [2]. Several physical factors affect seed germination, such as light,

\footnotetext{
*Correspondence: paola.vittorioso@uniroma1.it

${ }^{\dagger}$ Equal contributors

'Istituto Pasteur Fondazione Cenci Bolognetti, Dipartimento di Biologia e Biotecnologie "C. Darwin", Sapienza Università di Roma, Piazzale Aldo Moro 5, 00185 Rome, Italy

${ }^{3}$ Dipartimento di Biologia e Biotecnologie "C. Darwin", Sapienza Università di Roma, Piazzale Aldo Moro 5, 00185 Rome, Italy

Full list of author information is available at the end of the article
}

temperature and water potential. The effect of light is mediated mainly by the photoreceptor phytochrome B (phyB) [3], and the levels of GA and ABA are oppositely modulated by light, which induces GA biosynthesis and causes a reduction in $\mathrm{ABA}$ levels $[4,5]$. Among the regulators involved in phyB-mediated GA-induced seed germination in Arabidopsis, the bHLH transcription factor PHYTOCHROME INTERACTING FACTOR 3-LIKE 5 (PIL5) represents the master repressor [6]. In seeds kept in the darkness, PIL5 activates transcription of GAINSENSITIVE (GAI) and REPRESSOR OF ga1-3 (RGA) [7], two nuclear-localized DELLA transcriptional regulators that repress GA-mediated responses and are rapidly degraded in response to GA [8-10]. Indeed, it has been shown that in Arabidopsis all DELLA proteins are under 
negative control by GA and the proteasome [11]. Accordingly, gain-of-function della mutants show GA-insensitive phenotypes (i.e. dwarfism), whereas loss-of-function mutations result in GA-hypersensitive phenotypes (e.g. increased height) [12].

The DELLA proteins represent a subfamily of the GRAS plant transcription factors, and are characterized by the $\mathrm{N}$-terminal DELLA domain. In Arabidopsis there are five $D E L L A$ genes: the above mentioned GAI and RGA, and RGA-LIKE 1,2,3 (RGL 1,2,3). An insertional mutagenesis approach enabled cloning of Arabidopsis $G A I$ by isolation of a Ds transposon-mutated gai-t6 allele [13], while $R G A$ was identified by loss-of-function mutations [14] and shown to encode a protein closely related to GAI [15]. GAI and RGA were shown to have overlapping functions in repressing many growth processes, such as leaf expansion, stem elongation, floral initiation and seed germination [16,17]. Moreover, double mutant seeds have a higher germination rate than the wild-type ones in response to increasing Red (R) light fluences [7].

As of other DELLA proteins involved in seed germination, RGL2 also plays a negative key role: genetic data clearly showed that only a combination of $r g a$ and $r g l 2$ or gai-t6 and rgl2 mutant alleles could restore seed germination in a ga1-3 background [18].

We have previously shown that the DOF transcription factor DAG1 (DOF AFFECTING GERMINATION1) is a repressor of seed germination in Arabidopsis: dag1 knockout mutant seeds require lower GA and R light fluence rates than wild-type seeds to germinate [19-21]. We have also pointed out that DAG1 acts in the phyB-mediated pathway: DAG1 expression is reduced in seeds irradiated for 24 hours with $\mathrm{R}$ light, and this reduction is dependent on PIL5; in pil5 mutant seeds DAG1 expression is reduced irrespective of light conditions, indicating that DAG1 acts downstream of PIL5; moreover, DAG1 negatively regulates GA biosynthesis by directly repressing the GA biosynthetic gene AtGA3ox1 [22]. Very recently, we demonstrated that GAI cooperates with DAG1 in repressing AtGA3ox1, and that it directly interacts with DAG1 [23].

In order to further clarify the role of DAG1 in phyBmediated seed germination, we focus here on the functional relationship between DAG1, RGA and GAI in the control of this process. We provide genetic and phenotypic evidence suggesting different roles of the two DELLA proteins in seed germination and with respect to DAG1.

\section{Results}

The gai-t6 and rga28 mutant alleles show different seed germination phenotypes

It has been reported that concurrent inactivation of both GAI and RGA increases the seed germination potential: gai-t6rga28 double mutant seeds require less $\mathrm{R}$ light fluences than wild-type ones to germinate [7] - a phenotype that is reminiscent of dag1 mutant seeds, which need a fluence rate six times lower than wild-type to germinate [20]. We compared the seed germination properties of stored (28 days after ripening, DAR) gai-t6, rga28, double mutant gai-t6rga28 and Col-0 wild-type seeds, under phyB-dependent germination conditions [7,22]. We also assessed the germination properties under white light and in the dark, with or without stratification.

Under phyB-dependent conditions, in the absence of stratification, germination rate of rga28 mutant seeds (28 DAR) was only 38\%, compared with almost $100 \%$ of gai-t6 and gai-t6rga28 seeds and of wild-type seeds. Instead, after stratification, all mutant lines and wild-type seeds germinated completely (Figure 1A).

Under white light the only substantial difference in the germination rate of stratified seeds was observed at 24 hours between rga28 and gai-t6rga 28 mutant seeds compared to wild-type ones (56\%, 64\% and $85 \%$ respectively), and in all cases $100 \%$ germination was attained in 72 hours (Figure 1B). In the absence of cold treatment, although all lines reached 100\% germination after 96 hours, gai-t6 seeds germinated faster and rga28 seeds slower than wildtype, while gai-t6rga28 mutants showed the same germination kinetics of the latter, i.e. roughly $60 \%, 40 \%$ and $30 \%$, respectively, after 24 hours (Figure $1 C$ ). After 5 days in the dark, stratified seeds of all mutant lines germinated completely as did wild-type seeds; on the contrary, without stratification, the germination rate of gai-t6 and wild-type seeds were similar (above 80\%), whereas both rga28 and gai-t6rga28 seeds germinated significantly less (approximately $40 \%$ ) (Figure 1D). As one function of stratification is to remove seed dormancy, we verified whether the rga28 germination phenotype was due to increased seed dormancy.

A seed germination assay, without stratification, was performed with freshly harvested mutant seeds, and with seeds respectively at 7, 14, 21 DAR, to asses a possible loss of dormancy due to seed storage. The germination rate was scored after seven days under white light. Freshly harvested and 7 DAR single gai-t6 and rga28 and double gai-t6rga28 mutant seeds showed a germination rate lower than $10 \%$, similarly to wild-type seeds ( $8 \%$ germination). The germination of gai-t6 and wild-type seeds increased up to $48 \%$ and $52 \%$, respectively, after two weeks of storage; dormancy was almost completely relieved after three weeks $83 \%$ and $97 \%$ germination for gai-t6 and wild-type seeds, respectively. Conversely, rga28 and gai-t6rga28 14 DAR seeds still retained a significantly higher level of dormancy, as revealed by a germination rate of $30 \%$ and $22 \%$, respectively. After three weeks of storage both rga28 and gai-t6rga28 mutant seeds lost part of their dormancy (38\% and 53\% germination, respectively), although only $\mathrm{rga} 28$ seeds showed a significant difference with wild-type seeds (97\% germination) (Figure 1E). 


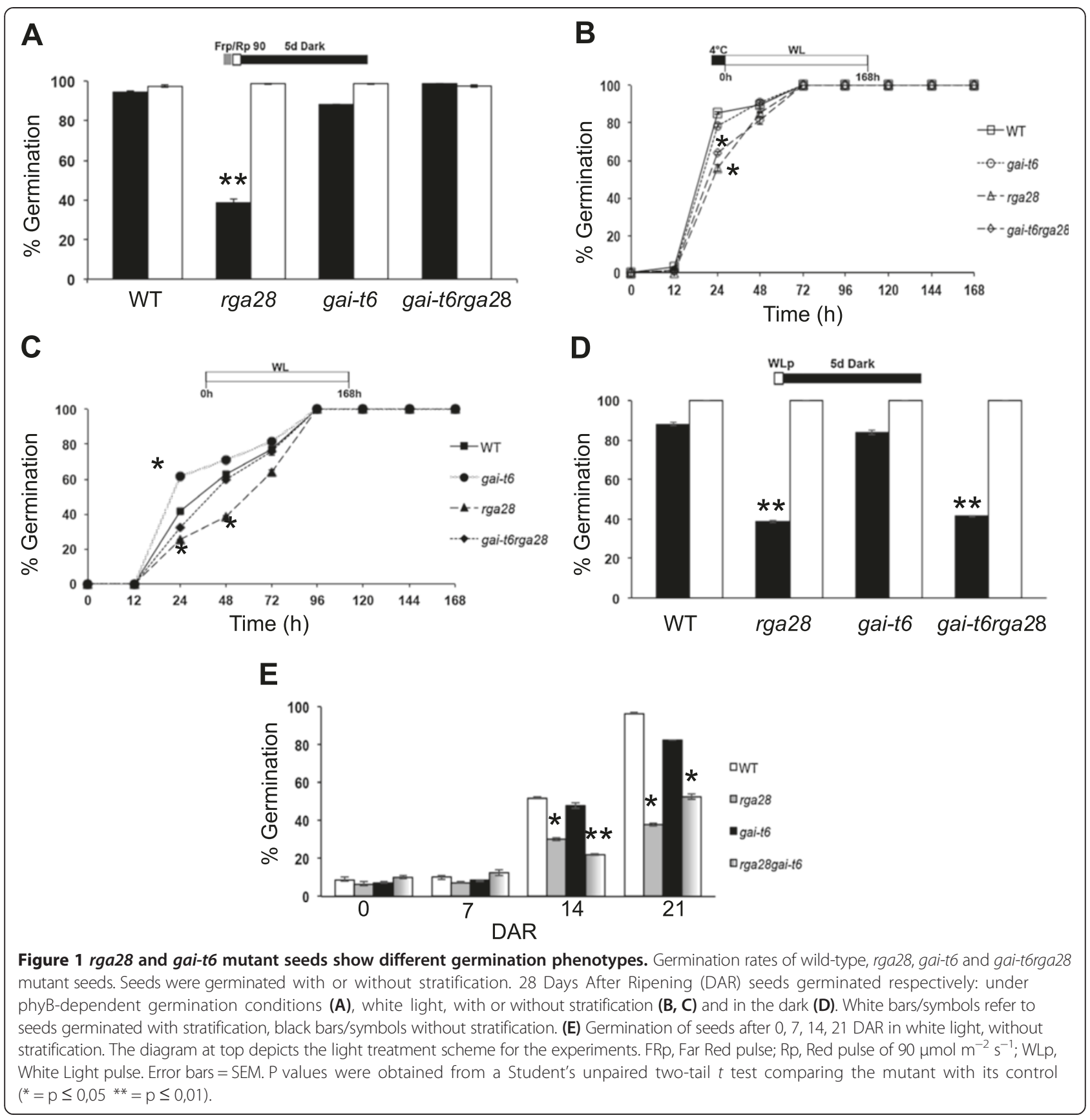

These results point to different effects of GAI and RGA on seed dormancy: the absence of RGA severely increases dormancy, while lack of GAI partially compensates $R G A$ inactivation, as gai-t6rga 28 mutant seeds show a milder phenotye than the rga28 single mutant.

\section{The dag1 and rga28 mutations are not epistatic}

To elucidate the genetic relationship between the DOF gene DAG1 and the DELLA-encoding genes RGA and GAI, we constructed the dag1rga28 double mutant. In contrast, attempts to isolate the dag1gai-t6 double mutant were unsuccessful (see below). As the dag1 and rga28 mutant lines are in different ecotypes (Ws- 4 and Col-0, respectively), several lines for each genotype - double mutants, parental lines and wild-type - were selected and analysed in order to minimize the effect of the ecotype on the phenotype of interest.

Seed germination assays under phyB-dependent conditions (i.e. after exposure to a pulse of $\mathrm{R}$ light) revealed that, in the absence of stratification, the germination rate of dag1rga28 double mutant seeds was similar to wildtype seeds (58\% and 56\% respectively), whereas the dag1 
and rga28 single mutant seeds had significant different germination rates (68\% and 39\% respectively), compared to wild-type. In contrast, upon stratification all mutant lines and wild-type seeds germinated almost completely (Figure 2A). After stratification and under white light, $100 \%$ germination was attained in 72 hours by mutant and wild-type seeds, although $\mathrm{rga} 28$ mutant seeds showed a significative slower kinetics (56\% at 24 hours, compared to 85,89 and $91 \%$, respectively, of wild-type, dag1 and dag1rga28) (Figure 2B). Under white light without stratification, rga28 seeds exhibit germination properties significantly lower (25\%) than dag1rga28 (31\%), wild-type (41\%) and dag1 (45\%) seeds as measured at 24 hours (Figure 2C). After 5 days in the dark, stratified seeds of the mutant lines germinated completely as wild-type seeds (Figure 2D); on the contrary, in the absence of stratification, wild-type, dag1 and dag1rga28 double mutant seeds showed similarly high germination rates (88\%, 85\% and $83 \%$, respectively), whereas $\mathrm{rga} 28$ seeds displayed a significantly lower germination percentage (39\%) (Figure 2D).

Since the dag1rga28 seed germination phenotype is not completely similar to that of the single mutants, dag1 and rga28 do not have an epistatic relationship.
Simultaneous inactivation of both DAG1 and GAl affects embryo development

As for the dag1gai-t6 double mutant, we analysed by PCR-based genotyping more than one hundred F2 plants derived from both the dag1 $\times$ gai-t6 and the reciprocal cross, but we were unable to isolate the dag1gai-t6 double mutant. To verify the possibility that concurrent inactivation of both DAG1 and GAI may affect embryo development, we performed a macroscopic analysis of siliques from plants of the F1 generation, which contain F2 seeds segregating different combinations of wild-type and mutant alleles of both DAG1 and GAI (Figure 3A). We compared the F2 seeds derived from the crosses with those of dag1 and gai-t6 single mutant seeds and of their respective wild-type seeds. Moreover, as the single mutants are in different ecotypes, the F2 seeds were also compared with seeds in siliques derived from a Ws-4 $\times$ Col-0 cross, and with the parental lines (dag1, gai-t6) also derived from the dag1 $\times$ gai-t6 cross (Additional file 1: Figure S1). The results of this analysis revealed a high percentage of aborted seeds (35\%) in the F2 generation from the dag1 $\times$ gai-t6 and reciprocal crosses, compared with about $1 \%$ in the different wild-type siliques, including those from the Ws $-4 \times$ Col- 0 cross. Interestingly, while we

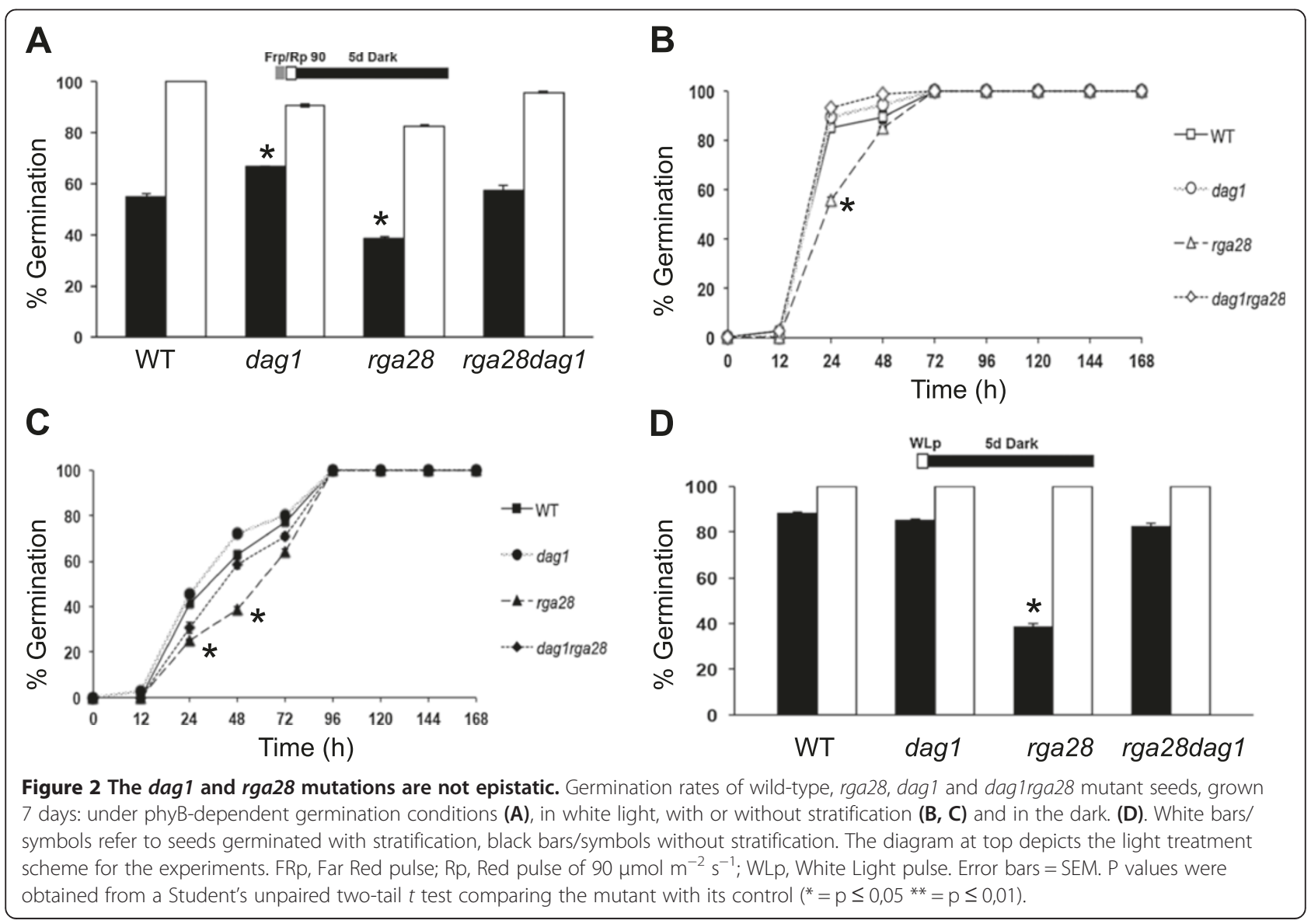




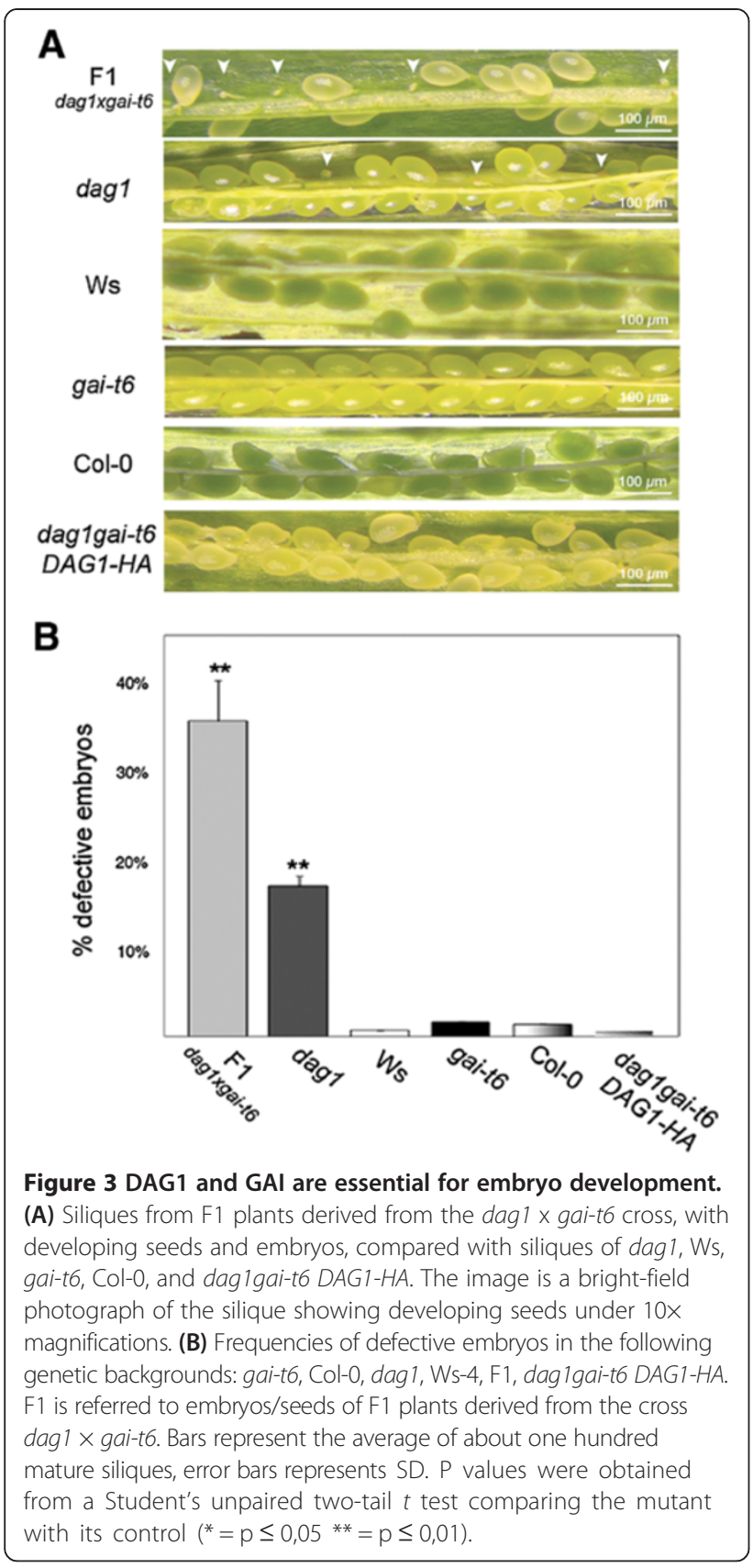

observed only $2 \%$ of aborted seeds in the siliques of the gai-t6 single mutant, siliques from the dag1 single mutant contained $17 \%$ of abnormal seeds, indicating that lack of DAG1 results in embryonic defects and that the simultaneous absence of GAI enhances this phenotype (Figure 3A,B). In addition, in order to minimize the possibility that the embryo-lethal phenotype could be due to the combination of dag1 with the gai-t6 allele in the Col-0 ecotype, we performed the same crosses with the gai-t6 allele in Ler background. Analysis by PCR-based genotyping of about one hundred F2 plants was again unsuccessful, as we could not isolate the dag1gai-t6 double mutant. Both the frequencies of the dag1 and gai-t6 single mutants and of the heterozygous lines were different from what expected (Figure 4). Further genetic analyses will be required to verify whether any of the different allelic combinations has viability and/or germination problems.

We then analyzed the phenotype of F2 embryos and checked for additional phenotypes compared to wildtype and single mutants. In wild-type, dag1 and gai-t6 single mutants, transversal division of basal vascular cells at globular stage led to asymmetric cells (Figure 5A, $\mathrm{C}, \mathrm{E}, \mathrm{G})$. In contrast, some F2 embryos displayed longitudinal divisions (Figure 5I,K), thus altering the radial symmetry of the embryo axis (Figure 5J,L) observed in control plants (Figure 5B,D,F,H). An additional phenotype was observed at the transition stage where individuals of the F2 embryos showed aberrant triangular shape, as highlighted by the arrow (Figure 5P) and also shown in the 3D image (Figure 5Q,R) compared to wild-type embryos (Figure 5M-O). Interestingly, a small percentage of dag1 embryos also showed similar phenotypes (Figure 6).

\section{Expression of DAG1 complements embryo defects and germination properties}

To verify whether expression of the DAG1-HA chimaeric protein would, at least in part, complement the abovedescribed embryo defects, we crossed the dag1DAG1-HA line with the gai-t6 single mutant. Out of 28 F2 plants derived from the cross, we were able to isolate seven dag1gai-t6DAG1-HA lines. Macroscopic analysis of siliques from these plants revealed normally-developing

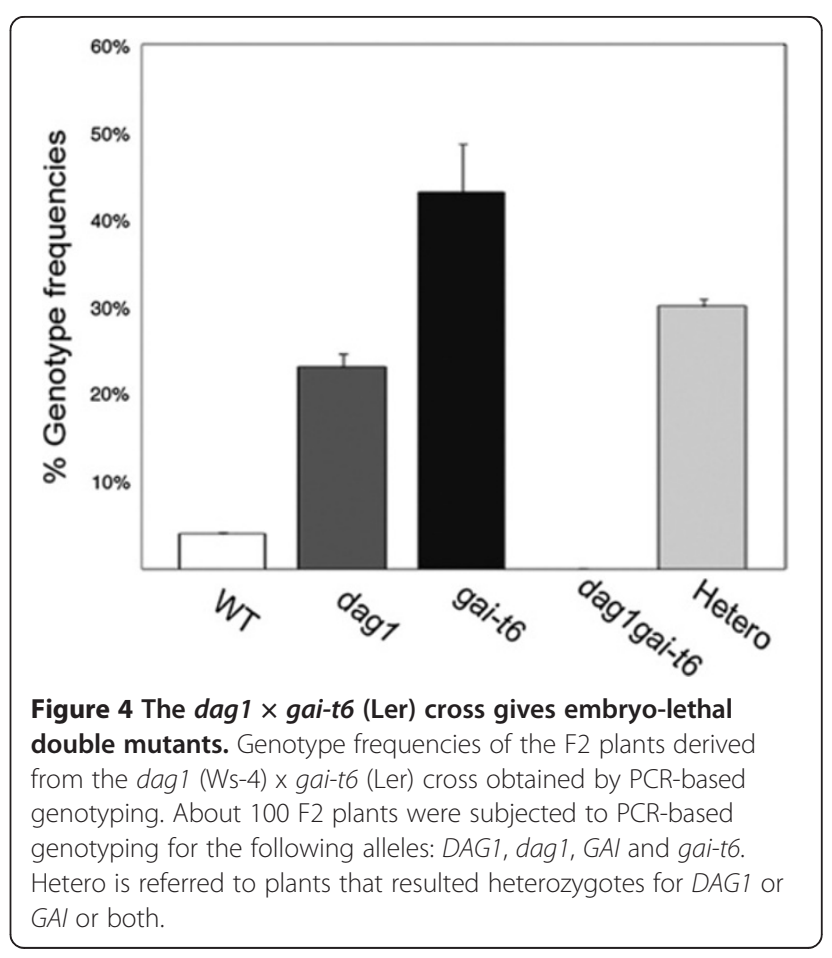




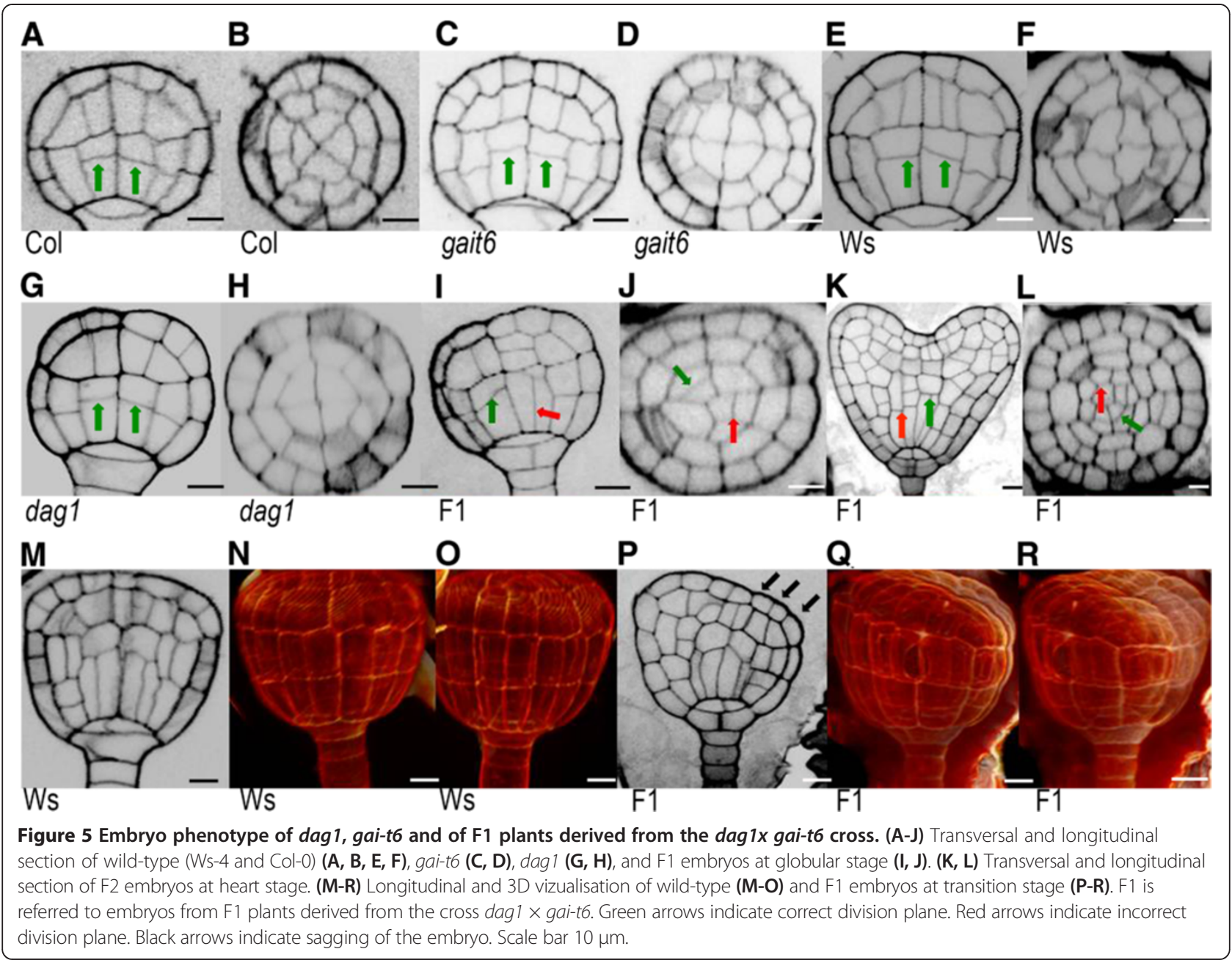

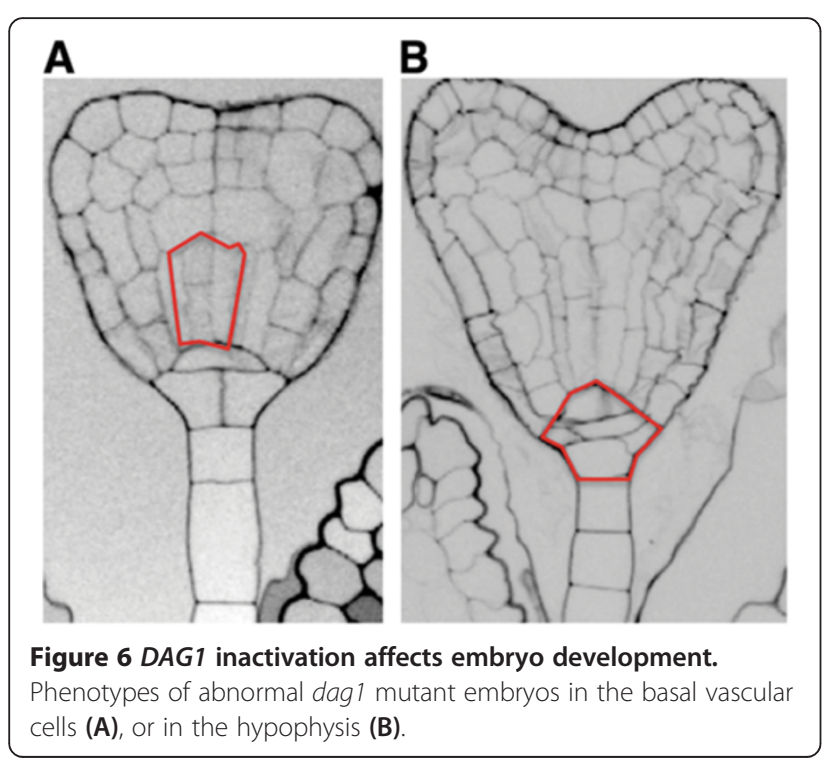

seeds with a percentage of aborted seeds similar to wildtype (Figure 3A,B). Moreover, we analysed the germination properties of dag1gai-t6DAG1-HA seeds, as well as of dag1DAG1-HA seeds, under phyB-dependent germination conditions, and in the presence or absence of stratification both under white light and in the dark (Figure 7). The transgenic lines were compared with the corresponding wild-type (Ws and Ws/Col respectively for dag1DAG1-HA and dag1gai-t6DAG1-HA). Under all conditions tested, the germination rates of these transgenic lines were not significantly different from those of wild-type seeds. The only conspicuous difference regarded dag1DAG1-HA seeds which germinated significantly slower than wild-type (Figure 7B,C).

\section{DAG1 is expressed during embryo development}

We have previously shown that DAG1 expression is localized in the vascular system of the plant. The DAG1 promoter is also active in the vascular tissue of seeds during the process of imbibition [21,22]. The involvement of DAG1 in the process of embryogenesis prompted us to 


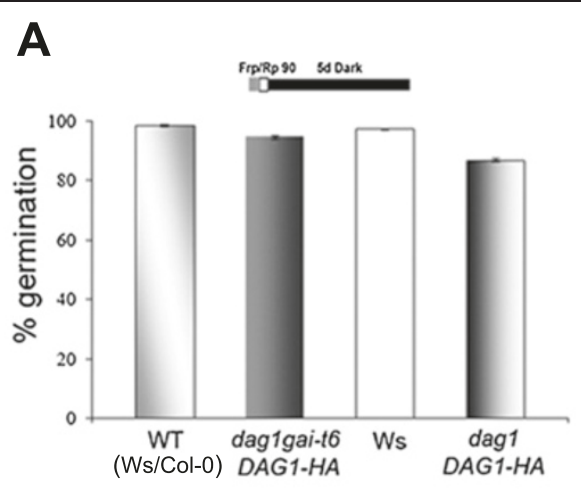

B

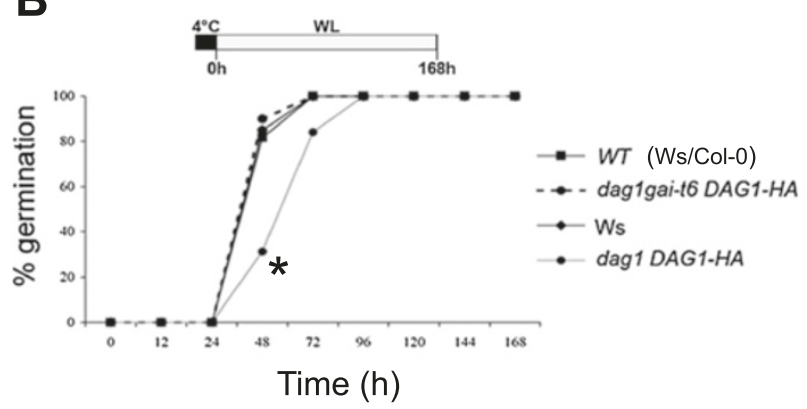

D

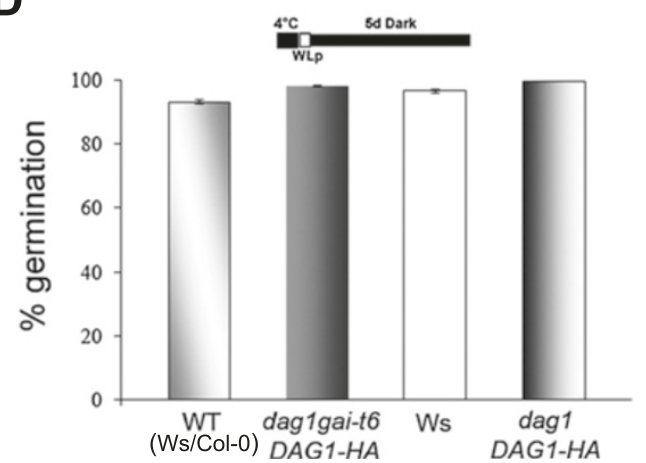

C

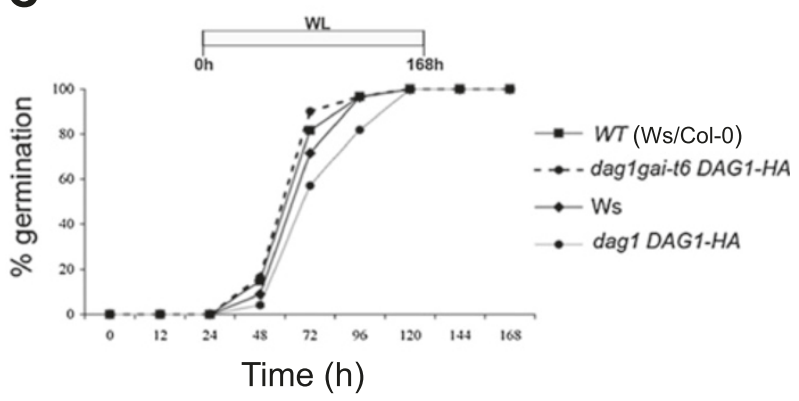

E

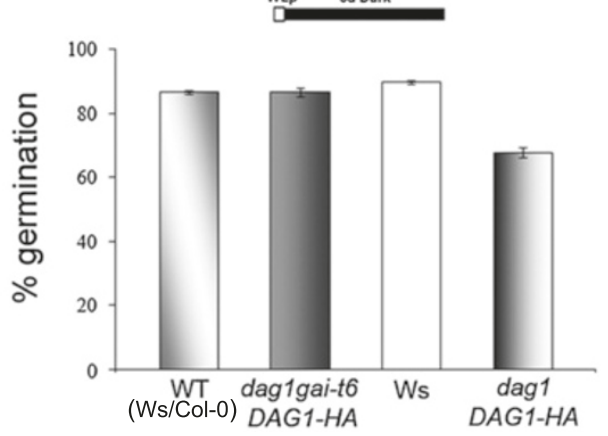

Figure 7 Overexpression of DAG1-HA complements the embryo mutant phenotype. Germination assays of dag1DAG1-HA and dag1gai-t6DAG1-HA seeds (28 DAR) and wild-type (Ws/Col-0), under phyB-dependent germination conditions (A), in white light (B, C) and in the dark. (D, E) with (B, D) or without stratification $(\mathbf{C}, \mathbf{E})$. Error bars $=$ SEM. P values were obtained from a Student's unpaired two-tail $t$ test comparing the mutant with its control $\left(^{*}=p \leq 0,05^{* *}=p \leq 0,01\right)$.

further analyse $D A G 1$ expression during embryo development. We used a DAG1:GUS reporter transgenic line utilized in a previous study [21]. GUS activity was observed in embryos at the globular, heart, torpedo, and bent cotyledon stages. Interestingly, GUS staining was extended to all cells at the globular stage, whereas from the heart stage on it was restricted to the procambium (Figure 8).

\section{Discussion}

We had previously characterized the DAG1 transcription factor as a repressor of seed germination [19-21] that acts downstream of PIL5 and negatively regulates GA biosynthesis [22]. As also the DELLA proteins RGA and GAI act downstream of PIL5 in seed germination [7], we investigated on the respective roles of these DELLA proteins in this process and their relationship with DAG1.

\section{RGA and GAI have distinct roles in seed germination}

RGA and GAI have been reported to be involved in several growth processes [16,17]; however, the single null mutants rga24, rga28 and gai-t6 were reported to lack any visible phenotype, and a functional redundancy of the two proteins had been suggested $[8,13,16]$. As for seed germination, the single rga28 and gai-t6 mutants were shown to behave similarly to the wild-type in response to increasing red light fluences [7].

Here we show that the rga28 and gai-t6 single mutants have different seed germination phenotypes, suggesting 

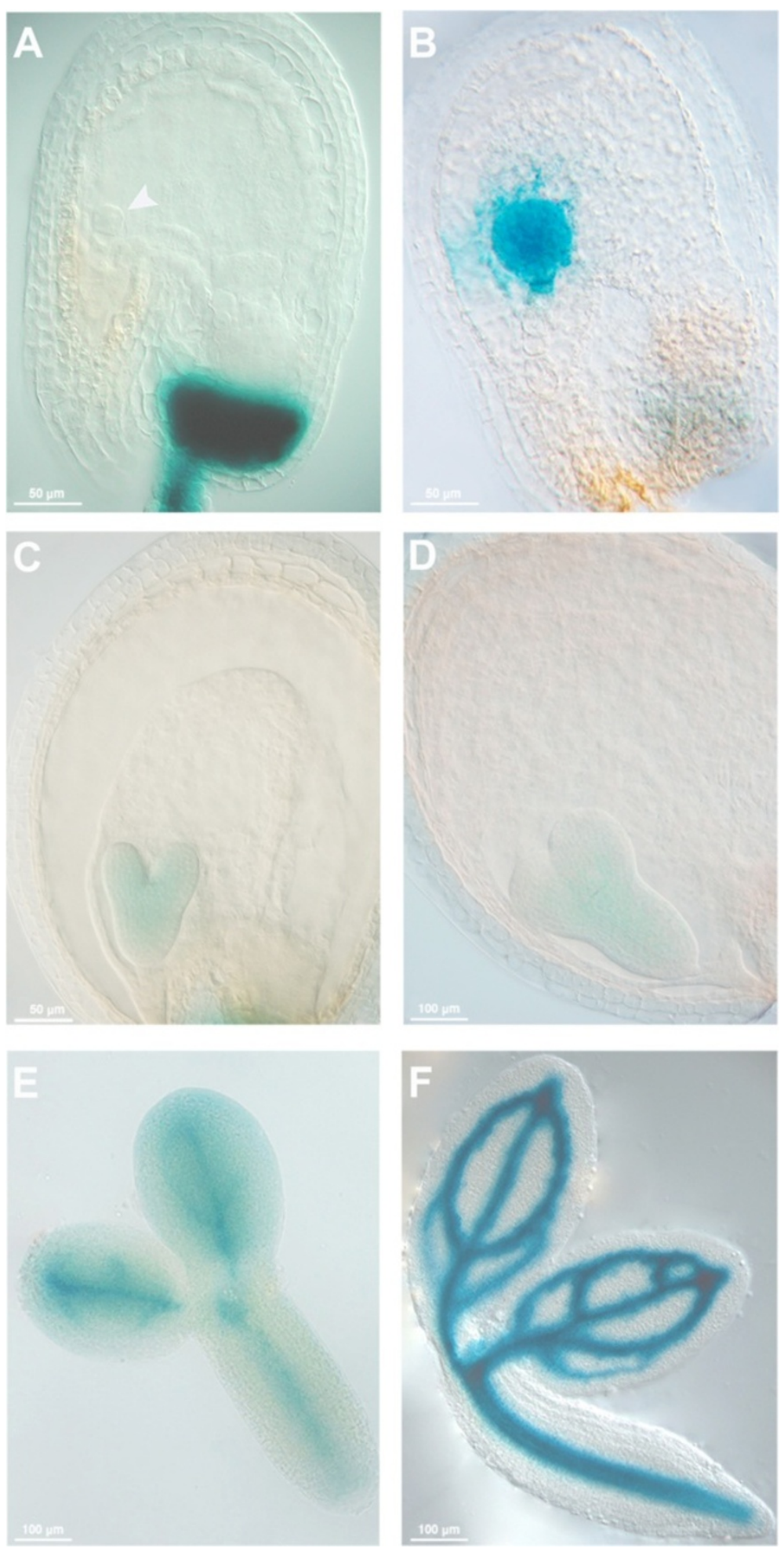

Figure 8 DAG1 is expressed during embryo development. Histochemical staining of DAG1:GUS during embryogenesis, in early globular, globular, heart, late heart, torpedo and mature embryo (A-F). Arrowhead in (A) indicates the embryo. 
(at least partially) distinct functions for RGA and GAI in this developmental process. In particular, rga28 seeds have, in the absence of stratification, a lower germination rate than wild-type irrespective of light conditions. This germination phenotype is likely due to an increased dormancy - as revealed by our germination assays on freshly harvested seeds and on seeds at different DAR.

Our data suggest that RGA plays a negative role in the regulation of seed dormancy. RGA has been shown to be involved in seed dormancy and to be directly activated by SPATULA (SPT), which also inhibits the negative regulator of RGA MOTHER OF-FT-AND-TFL1 (MFT) [24-26], but dormancy of the rga28 single mutant was not analysed by those authors.

On the other hand, our work shows that although the gai-t6 single mutant does not have a dormancy phenotype, lack of GAI partially compensates $R G A$ inactivation, as gai-t6rga28 mutant seeds show a milder phenotye than the rga28 single mutant. In addition, in our hands gai-t6 mutant seeds showed a germination potential slightly higher than wild-type under white light in the absence of stratification, similar to that of the dag1 mutant ([19]; this work).

It should be pointed out that $R G A$ and $G A I$ also differ in their transcriptional regulation in connection with DAG1: while we have recently shown a reciprocal negative transcriptional control of the genes DAG1 and GAI during seed germination [23], a previous microarray analysis of ours showed that $G A I$, but not $R G A$, was upregulated by $D A G 1$ inactivation [27].

\section{Inactivation of GAl enhances the dag1 embryo mutant phenotype}

We have previously reported that dag1 siliques contain numerous aborted seeds [19]. In this work, attempts to isolate the dag1gai-t6 double mutant were unsuccessful, suggesting that the simultaneous inactivation of both $D A G 1$ and $G A I$ results in an embryo-lethal phenotype, i.e. a more severe phenotype than inactivation of only $D A G 1$. This is not due to an additive effect of the two mutations, since a statistical analysis of the siliques revealed that while dag1 contained 17\% abnormal seeds, only $2 \%$ aborted seeds were present in gai-t6 and in wildtype siliques. Thus, the absence of GAI does not in itself lead to seed abnormalities, but inactivation of this gene in a dag1 mutant background is apparently responsible for embryo lethality. This may be an additional indication of the cooperation between DAG1 and GAI in controlling common target genes that we pointed out in a previous paper, where we showed that the two proteins cooperate in negatively regulating the $A t G A 3 o x 1$ gene [23]. Consistently, we could restore embryo development by expressing the DAG1-HA chimaeric protein in the dag1gai-t6 double mutant background.
The earliest phenotype of the dag1gai-t6 double mutant is an impairment in cell divisions in the basal portion of the globular stage embryo, the hypophyseal and the procambial precursor cells, but not in the ground precursor cells. Consistent with this mutant phenotype, the DAG1 promoter is active in the embryo starting from the globular stage.

Simultaneous inactivation of POLTERGEIST (POL) and POLTERGEIST-LIKE 1 (PLL1) results in defects in basal embryo patterning similar to what described here for the dag1gai-t6 double mutant [28]. POL and PLL1 are two related phosphatases required to establish the vascular axis in the embryo, by inducing expression of the WUSCHEL (WUS) homolog WUSCHEL RELATED HOMEOBOX 5 (WOX5).

It is tempting to speculate that DAG1 and GAI may also function in this molecular network. As the double mutant dag1gai-t6 has a more severe phenotype than the double mutant polpll1, one might hypothesize that DAG1 and GAI act upstream of POL and PLL1. Further analysis on the functional and molecular relationship among these factors will help unveiling the complex signaling underlying embryo development.

\section{Conclusions}

Here we show that the DELLA proteins RGA and GAI have, at least partially, different roles in the seed germination process. Indeed, $R G A$ inactivation results in increased seed dormancy, whereas lack of GAI partially compensates this phenotype, as gai-t6rga28 mutant seeds show a milder phenotye than the rga28 single mutant.

With respect to DAG1, our data suggest that this latter and RGA act in independent branches of the PIL5controlled germination pathway, whereas GAI and DAG1 are involved in embryo development since the dag1gai-t6 double mutant proved embryo-lethal. This latter finding should be regarded in the context of the cooperation of DAG1 and GAI in regulating common target genes, such as in the case of the GA biosynthetic gene AtGA3ox1 that we have very recently demonstrated [23].

\section{Methods}

\section{Plant material and growth conditions}

dag1 is the allele described in Papi et al. [19] in Ws-4 ecotype. The rga28, gai-t6 and gai-t6rga28 (Col-0) mutants, kindly provided by Dr. G. Choi, are described by Oh et al. [7]. dag1rga28 was obtained by crossing the single mutants, and identified in the F3 generation by PCR analysis. The gai-t6 and dag1 mutants were crossed using both lines as female parent. F1 plants derived from the cross were analysed by PCR to confirm the presence of the mutant alleles in heterozygosis. As the single mutants were in different ecotypes, the parental lines (dag1, rga28, gai-t6) and the wild-type were also selected from the cross. 
Several lines for each genotype were selected and analysed in order to minimize the effect of the two different ecotypes on the phenotypes of interest. The rga24 and gai-t6 mutant lines in Ler ecotype [14] were from the ABRC stock.

The dag1gai-t6DAG1-HA lines were isolated from the F2 generation derived from the cross gai-t6 $\times$ dag1DAG1-HA, by PCR-based genotyping.

All Arabidopsis thaliana lines used in this work were grown in a growth chamber at $24 / 21 \% \mathrm{C}$ with $16 / 8$-h day/night cycles and light intensity of $300 \mu \mathrm{mol} / \mathrm{m}^{-2} \mathrm{~s}^{-1}$ as previously described $[19,22]$. All the primers used for the screenings are listed in Additional file 2: Table S1.

\section{Seed germination assays}

All seeds used for germination tests were harvested from mature plants grown at the same time, in the same conditions, and stored for the same time $(7,14,21,28$ DAR) under the same conditions, except where freshly harvested seeds were used. Germination assays were performed according to Gabriele et al. [22]. For phyB-dependent germination experiments, seeds, with or without cold treatment (stratification, 2 days at $4^{\circ} \mathrm{C}$ ), were exposed to a pulse of FR light $\left(40 \mu \mathrm{mol} \mathrm{m}^{-2} \mathrm{~s}^{-1}\right)$, then a pulse of R light $\left(90 \mu \mathrm{mol} \mathrm{m} \mathrm{m}^{-2} \mathrm{~s}^{-1}\right)$ and subsequently kept in the dark for 5 days: under these conditions germination is mediated only by phyB. For the germination assays in the dark, seeds were exposed to a pulse of white light, then kept in the dark for 5 days. All germination assays were repeated with three seed batches, and one representative experiment is shown. Bars represent the mean \pm SEM of three biological repeats (25 seeds per biological repeat). P values were obtained from a Student's unpaired two-tail $t$ test comparing the mutant with its control $(*=\mathrm{p} \leq 0,05 * *$ $\mathrm{p} \leq 0,01)$.

\section{Cytology and microscopy}

For staining of ovules and seeds, siliques were harvested and slit open on one side. Tissue was fixed in 50\% methanol/10\% acetic acid and then subjected to $3 \mathrm{~h}$ treatment of $1 \%$ SDS and $0.2 \mathrm{~N} \mathrm{NaOH}$ at room temperature. Siliques were rinsed in water, incubated in $25 \%$ bleach solution $\left(2.5 \%\right.$ active $\left.\mathrm{Cl}^{-}\right)$for 1 to $5 \mathrm{~min}$, rinsed again, and then transferred to $1 \%$ periodic acid. The samples were then further processed as described before [29].

For confocal microscopy, a LSM 710 (Zeiss) spectral confocal laser-scanning microscope was used. Excitation wavelengths for propidium iodide-stained samples was $488 \mathrm{~nm}$. Data were processed for some two-dimensional orthogonal sections, 3D rendering, using the open source software Osirix ([30]; http://www.osirix-viewer.com/About OsiriX.html) on a quadxeon 2.66-Ghz, 2-GB RAM Apple Mac pro workstation.
Analysis of defective embryos of the F1 plants derived from the cross dag1 $\times$ gai-t6. was performed under an Axioskop 2 plus microscope (Zeiss). Bars represent the average of about one hundred mature siliques, error bars represents SD. P values were obtained from a Student's unpaired two-tail $t$ test comparing the mutant with its control $(*=\mathrm{p} \leq 0,05 * * \mathrm{p} \leq 0,01)$.

\section{GUS constructs and analysis}

The DAG1:GUS line is the one described in Gualberti et al. [21]. Histochemical staining and microscopic analysis were carried out according to Blazquez et al. [31]. Stained embryos (after washing in 70\% ethanol) were analysed and photographed under an Axioskop 2 plus microscope (Zeiss).

\section{Additional files}

Additional file 1: Figure S1. Analysis of defective embryos in the hybrid wild-type, F1, dag1, gai-t6 lines (Ws-4/Col-0). Bars represent the average of about one hundred mature siliques, error bars represents SD. $P$ values were obtained from a Student's unpaired two-tail $t$ test comparing the mutant with its control $\left({ }^{*}=p \leq 0,05^{* *}=p \leq 0,01\right)$.

Additional file 2: Table S1. List of the primers used for the screenings of the double mutants, and the isolation of the dag1gai-t6DAG1-HA transgenic line.

\section{Competing interests}

The authors declare that they have no competing interests.

\section{Authors' contributions}

PV designed the research. $A B$ and SS contributed to the experimental design and to analysis of the results. $A B, S S, D C, R L$ and $E M$ performed the experiments. KB and JCP performed microscopic analyses of the gai-t6dag 1 double mutant embryos. All authors analyzed and discussed the data. $A B$ and SS prepared the figures and PV wrote the article. PC supervised the research and the writing of the manuscript. All Authors read and approved the final manuscript.

\section{Acknowledgments}

We thank G. Choi who kindly provided the rga28, gai-t6, gai-t6rga28 mutant lines. This work was partially supported by research grants from Ministero dell'Istruzione, Università e Ricerca, Progetti di Ricerca di Interesse Nazionale, and from Sapienza Università di Roma to PC, and from Istituto Pasteur Fondazione Cenci Bolognetti to PV.

\section{Author details}

${ }^{1}$ Istituto Pasteur Fondazione Cenci Bolognetti, Dipartimento di Biologia e Biotecnologie "C. Darwin", Sapienza Università di Roma, Piazzale Aldo Moro 5, 00185 Rome, Italy. ${ }^{2}$ Institut Jean-Pierre Bourgin, UMR1318

INRA-AgroParisTech, Bâtiment 2, INRA, Centre de Versailles-Grignon, Route de St-Cyr (RD10), 78026 Versailles Cedex, France. ${ }^{3}$ Dipartimento di Biologia e Biotecnologie "C. Darwin", Sapienza Università di Roma, Piazzale Aldo Moro 5, 00185 Rome, Italy.

Received: 24 April 2014 Accepted: 16 July 2014

Published: 26 July 2014

\section{References}

1. Koornneef M, Karssen CM: Seed Dormancy and Germination in Arabidopsis. Cold Spring Harbor Laboratory Press: Cold Spring Harbor; 1994:313-333.

2. Koornneef $M$, Bentsink $L$, Hilhorst $H$ : Seed dormancy and germination. Curr. Opin. Plant Biol. 2002, 5:33-36. 
3. Shinomura T, Nagatani A, Chory J, Furuya M: The induction of seed germination in Arabidopsis thaliana is regulated principally by phytochrome B and secondarily by phytochrome A. Plant Physiol. 1994, 104:363-371.

4. Yamaguchi S, Smith MW, Brown RGS, Kamiya Y, Sun TP: Phytochrome regulation and differential expression of gibberellin $3 \beta$-hydroxylase genes in germinating Arabidopsis seeds. Plant Cell 1998, 10:2115-2126.

5. Seo M, Hanada A, Kuwahara A, Endo A, Okamoto M, Yamauchi $Y$, North $H$, Marion-Poll A, Sun TP, Koshiba T, Koshiba T, Kamiya Y, Yamaguchi S, Nambara E: Regulation of hormone metabolism in Arabidopsis seeds: phytochrome regulation of abscisic acid metabolism and abscisic acid regulation of gibberellin metabolism. Plant J. 2006, 48:354-366.

6. Oh E, Kim J, Park E, Kim Jl, Kang C, Choi G: PIL5, a phytochromeinteracting basic helix-loop-helix protein, is a key negative regulator of seed germination in Arabidopsis thaliana. Plant Cell 2004, 16:3045-3058.

7. Oh E, Yamaguchi S, Hu J, Yusuke J, Jung B, Paik I, Lee HS, Sun TP, Kamiya Y, Choi G: PIL5, a phytochrome-interacting bHLH protein, regulates gibberellin responsiveness by binding directly to the GAI and RGA promoters in Arabidopsis seeds. Plant Cell 2007, 19:1192-1208.

8. Tyler L, Thomas SG, Hu J, Dill A, Alonso JM, Ecker JR, Sun TP: DELLA proteins and gibberellin-regulated seed germination and floral development in Arabidopsis. Plant Physiol. 2004, 135:1008-1019.

9. Fleet CM, Sun TP: A DELLAcate balance: the role of gibberellin in plant morphogenesis. Curr Opin Plant Biol. 2005, 8:77-85.

10. Sun TP: The molecular mechanism and evolution of the GA-GID1-DELLA signaling module in plants. Curr Biol. 2011, 21:338-345.

11. Feng S, Martinez C, Gusmaroli G, Wang Y, Zhou J, Wang F, Chen L, Yu L, Iglesias-Pedraz JM, Kircher S, Schäfer E, Fu X, Fan LM, Deng XW: Coordinated regulation of Arabidopsis thaliana development by light and gibberellins. Nature. 2008, 451:475-479.

12. Ariizumi T, Hauvermale A, Nelson SK, Hanada A, Yamaguchi S, Steber CM: Lifting DELLA Repression of Arabidopsis Seed Germination by Nonproteolytic Gibberellin Signaling. Plant Physiol. 2013, 162:2125-2139.

13. Peng J, Carol P, Richards DE, King KE, Cowling RJ, Murphy GP, Harberd NP: The Arabidopsis GAl gene defines a signalling pathway that negatively regulates gibberellin responses. Genes Dev. 1997, 116:3194-3205.

14. Silverstone AL, Mak PY, Martínez EC, Sun TP: The new RGA locus encodes a negative regulator of gibberellin response in Arabidopsis thaliana. Genetics 1997, 146:1087-1099.

15. Silverstone AL, Ciampaglio CN, Sun TP: The Arabidopsis RGA gene encodes a transcriptional regulator repressing the gibberellin signal transduction pathway. Plant Cell 1998, 10(2):155-169.

16. Dill A, Sun TP: Synergistic derepression of gibberellin signaling by removing RGA and GAl function in Arabidopsis thaliana. Genetics 2001 159:777-785.

17. King K, Moritz T, Harberd N: Gibberellins are not required for normal stem growth in Arabidopsis thaliana in the absence of GAI and RGA. Genetics 2001, 1596:767-776.

18. Cao D, Hussain A, Cheng H, Peng J: Loss of function of four DELLA genes leads to light- and gibberellin-independent seed germination in Arabidopsis. Planta 2005, 2236:105-113.

19. Papi M, Sabatini S, Bouchez D, Camilleri C, Costantino P, Vittorioso P: Identification and disruption of an Arabidopsis zinc finger gene controlling seed germination. Genes Dev. 2000, 14:28-33.

20. Papi M, Sabatini S, Altamura MM, Henning L, Schafer E, Costantino P, Vittorioso P: Inactivation of the phloem-specific DOF zinc finger gene DAG1 affects response to light and integrity of the testa of Arabidopsis seeds. Plant Physiol. 2002, 128:411-417.

21. Gualberti G, Papi M, Bellucci L, Ricci I, Bouchez D, Camilleri C, Costantino P, Vittorioso P: Mutations in the DOF zinc finger genes DAG1 and DAG2 influence with opposite effects the germination of Arabidopsis seeds. Plant Cell 2002, 14:1253-1263.

22. Gabriele S, Rizza A, Martone J, Circelli P, Costantino P, Vittorioso P: The DOF protein DAG1 mediates PIL5 activity on seed germination by negatively regulating the GA biosynthetic gene AtGA3ox1. Plant J. 2010, 61:312-323.

23. Boccaccini A, Santopolo S, Capauto D, Lorrai R, Minutello E, Serino G, Costantino P, Vittorioso P: The DOF protein DAG1 and the DELLA protein GAI cooperate in negatively regulating AtGA3ox1 gene. Molecular plant 2014 , in press.
24. Penfield S, Josse EM, Kannangara R, Gilday AD, Halliday KJ, Graham IA: Cold and light control seed germination through the bHLH transcription factor SPATULA. Curr. Biol. 2005, 15:1998-2006.

25. Penfield S, Gilday AD, Halliday KJ, Graham IA: DELLA-mediated cotyledon expansion breaks coat-imposed seed dormancy. Curr Biol 2006, 16(23):2366-2370

26. Vaistij FE, Gan Y, Penfield S, Gilday AD, Dave A, He Z, Josse EM, Choi G, Halliday KJ, Graham IA: Differential control of seed primary dormancy in Arabidopsis ecotypes by the transcription factor SPATULA Proc Natl Acad Sci U S A 2013, 110:10866-10871.

27. Rizza A, Boccaccini A, Lopez-Vidriero I, Costantino P, Vittorioso P: Inactivation of the ELIP1 and ELIP2 genes affects Arabidopsis seed germination. New Phytol. 2011, 190:896-905.

28. Song S-K, Hofhuis H, Min Lee M, Clark SE: Key divisions in the early Arabidopsis embryo require POL and PLL1 phosphatases to establish the root stem cell organizer and vascular axis. Develop. Cell 2008, 15:98-109.

29. Truernit E, Bauby H, Dubreucq B, Grandjean O, Runions J, Barthélémy J, Palauqui JC: High-resolution whole-mount imaging of three-dimensional tissue organization and gene expression enables the study of Phloem development and structure in Arabidopsis. Plant Cell. 2008, 20:1494-1503.

30. Rosset A, Spadola L, Ratib O: OsiriX: an open-source software for navigating in multidimensional DICOM images. J Digit Imaging. 2004, 17:205-216.

31. Blázquez MA, Soowal LN, Lee I, Weigel D: LEAFY expression and flower initiation in Arabidopsis. Development 1997, 124:3835-3844.

doi:10.1186/s12870-014-0200-z

Cite this article as: Boccaccini et al.: Independent and interactive effects of DOF affecting germination 1 (DAG1) and the Della proteins GA insensitive (GAI) and Repressor of ga1-3 (RGA) in embryo development and seed germination. BMC Plant Biology $2014: 200$.

\section{Submit your next manuscript to BioMed Central and take full advantage of:}

- Convenient online submission

- Thorough peer review

- No space constraints or color figure charges

- Immediate publication on acceptance

- Inclusion in PubMed, CAS, Scopus and Google Scholar

- Research which is freely available for redistribution 